\title{
Identification of Exogenous Nitric Oxide-Responsive miRNAs from Alfalfa (Medicago sativa L.) under Drought Stress by High-Throughput Sequencing
}

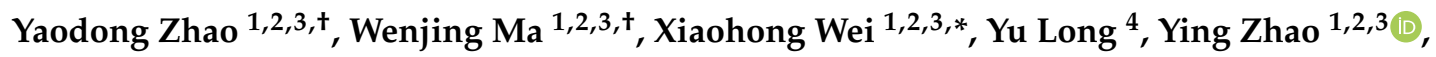 \\ Meifei Su ${ }^{1,2,3}$ and Qiaojuan Luo ${ }^{1,2,3}$ \\ 1 College of Life Science and Technology, Gansu Agricultural University, Lanzhou 730070, China; \\ zhaoyaodong927@163.com (Y.Z.); wenjingma24@163.com (W.M.); zhaoying924@outlook.com (Y.Z.); \\ sumeifei369@163.com (M.S.); 13919338538@163.com (Q.L.) \\ 2 Gansu Key Laboratory of Crop Genetic Improvement and Germplasm Innovation, Lanzhou 730070, China \\ Gansu Key Laboratory of Arid Habitat Crop Science, Lanzhou 730070, China \\ 4 College of Business Administration, Kent State University, Kent, OH 44240, USA; ylong@kent.edu \\ * Correspondence: weixh@gsau.edu.cn; Tel.: +86-138-9331-7951 \\ + These authors contribute equally to this work.
}

Received: 25 October 2019; Accepted: 24 December 2019; Published: 26 December 2019

\begin{abstract}
Alfalfa (Medicago sativa L.) is a high quality leguminous forage. Drought stress is one of the main factors that restrict the development of the alfalfa industry. High-throughput sequencing was used to analyze the microRNA (miRNA) profiles of alfalfa plants treated with CK (normal water), PEG (polyethylene glycol-6000; drought stress), and PEG + SNP (sodium nitroprusside; nitric oxide (NO) sprayed externally under drought stress). We identified 90 known miRNAs belonging to 46 families and predicted 177 new miRNAs. Real-time quantitative fluorescent PCR (qRT-PCR) was used to validate high-throughput expression analysis data. A total of 32 (14 known miRNAs and 18 new miRNAs) and 55 (24 known miRNAs and 31 new miRNAs) differentially expressed miRNAs were identified in PEG and PEG + SNP samples. This suggested that exogenous NO can induce more new miRNAs. The differentially expressed miRNA maturation sequences in the two treatment groups were targeted by 86 and 157 potential target genes, separately. The function of target genes was annotated by gene ontology (GO) enrichment and kyoto encyclopedia of genes and genomes (KEGG) analysis. The expression profiles of nine selected miRNAs and their target genes verified that their expression patterns were opposite. This study has documented that analysis of miRNA under PEG and PEG + SNP conditions provides important insights into the improvement of drought resistance of alfalfa by exogenous NO at the molecular level. This has important scientific value and practical significance for the improvement of plant drought resistance by exogenous NO.
\end{abstract}

Keywords: drought stress; alfalfa; exogenous nitric oxide; miRNAs; differential expression

\section{Introduction}

As a perennial forage, alfalfa has the advantages of good nutritional quality and high yield [1]. It originated in Asia and Transcaucasia [2]. Alfalfa has been widely planted all over the world, and has now been identified as a leading forage crop [3]. As a legume plant, alfalfa can improve the soil planting environment by fixing atmospheric nitrogen, also reducing the concentration of diethylhexyl phthalate (DEHP) in the soil, thereby protecting the ecological environment [4]. Furthermore, the root system of alfalfa can effectively prevent water loss and soil erosion [5]. With the increasing demand for feed, alfalfa has been planted all over the country. However, as a global habitat problem, drought has seriously affected the yield and quality of alfalfa and restricted the development of the alfalfa industry [6]. 
Therefore, it is imperative to improve the drought resistance of alfalfa. Recently, the application of exogenous materials is one of the main strategies involved in this alleviation of drought stress [7].

Nitric oxide (NO) is an important bioactive molecule in plants, which is catalyzed and synthesized by nitric oxide synthase (NOS) and nitrate reductase (NR) [8]. NO is involved in plant germination, root and leaf development, respiration, morphological occurrence, and delayed aging [9]. Studies have shown that the addition of exogenous SNP (sodium nitroprusside) can increase the content of endogenous NO in plants, which improves the resistance of plants under salinity [10], drought [11], heavy metal [12], heat [13], and cold damage stress [14]. NO not only directly causes changes in protein and enzyme activity, but also regulates gene transcription and post-translational modification, thereby enhancing the ability of plants to recover from drought stress [15]. However, studies conducted on improving drought resistance of alfalfa by exogenous NO mainly focus on physiological and biochemical aspects $[16,17]$, and have received less attention in molecule research, especially in terms of miRNA. The mechanism of exogenous NO enhancing the tolerance in alfalfa to drought stress has not been fully revealed.

miRNA is about $22 \mathrm{nt}$ long, and acts on the target gene and negatively regulates its expression level [18]. The miRNAs synthesized by the classical and non-canonical pathways can be complementary to miRNA and can be used to degrade the target gene or inhibit the translation of the target gene by binding to RISC (RNA-induced silencing complex) [19,20]. With the increasing maturity and development of small RNA sequencing, more and more miRNA sequences have been discovered and included. As of March 2018, a total of 38,589 precursor sequences and 48,885 mature sequences of 271 species were included in the miRNA database, which will provide an important tool for the study of miRNA. In the existing reports, miRNAs appear in many essential biological metabolic processes in plants, such as seed germination and signal transduction [21,22]. The function of some miRNAs has also been identified. For example, overexpression of miR172 can seriously affect flower development [23], conserved miR159 controls leaf morphogenesis, and miR396 regulates abscisic acid (ABA) expression $[24,25]$. However, the functions of some miRNAs have not all been identified. There exists a need for further investigation. Many miRNAs arise when plants cope with various stresses. Research reporting on miRNA involvement in some plant species' ability to cope with drought stress, such as tomato [26], potato [27], cotton [28], and Medicago truncatula [29], has been extensively studied. Due to the recent reports on the molecular mechanism of plant drought resistance, miRNA have garnered the interest of many experts and scholars.

Some drought-related miRNAs in leaves and roots of Medicago sativa 'Aohan' have been identified, and they have proven that miRNA plays an important role in regulating the drought resistance of alfalfa [30]. Muhammad Arshad et al. found that miR156 improved the drought resistance of alfalfa by acting on WD40-2 [31]. In addition, salinity-regulated [32], fall dormancy-related [33], freezing stress-related [34], and Pi deficiency-related [35] miRNAs have been identified in Medicago sativa. The above results suggest that miRNA plays a key role in the metabolism of alfalfa. This provides a theoretical basis and explains the exogenous nitric oxide-regulated miRNA expression in alfalfa drought resistance.

Because of exogenous NO function in the drought resistance of alfalfa and the potential value of miRNA in the stress resistance of alfalfa, it is boldly envisaged that exogenous NO regulates the expression of miRNA in alfalfa under drought stress. In our study, medium drought-tolerant variety Medicago sativa 'San deli' was used as the experimental material. Polyethylene glycol-6000 (PEG) was employed to simulate drought stress, along with spraying SNP (sodium nitroprusside, NO donor) externally. By analyzing the role of differentially expressed miRNA and their target sequence, the study aimed to (1) comprehensively screen the drought stress-responsive miRNA, (2) determine exogenous nitric oxide-regulated miRNAs under drought stress, and (3) make out the function of differentially expressed miRNA by analyzing the role of differentially expressed miRNA target genes. Exploring the mechanism of miRNA responding to $\mathrm{NO}$ under drought stress will provide a theoretical basis and technical support for the application of exogenous NO in plant stress resistance. 


\section{Materials and Methods}

\subsection{Plant Materials and Growth Conditions}

Medicago sativa L. 'Sandeli' was used in this study. Alfalfa plants were grown in pots with an a diameter of $15 \mathrm{~cm}$, containing a mixture of nutrient soil and vermiculite $(8: 2 \mathrm{v} / \mathrm{v})$, in a growth chamber at $25 \pm 1^{\circ} \mathrm{C}$ under a $12 \mathrm{~h}$ light and dark photoperiod. Plants were supplied with murashige-skoog (MS) nutrient solution. After alfalfa plants reached the age of 30 days, they were then randomly separated into three groups: CK (normal water), PEG (drought stress), and PEG + SNP (drought stress and SNP treatment) groups. Drought groups were conducted by watering $50 \mathrm{~mL}$ of $10 \%$ PEG solution once after every 2 days for 7 days. The PEG + SNP group sprayed $5 \mathrm{~mL}$ of $0.01 \mathrm{mmol} / \mathrm{L}$ SNP per day while drought was applied. Plants in the CK and PEG samples were simultaneously at 5 mL. Leaf samples from 30 plants in CK, PEG, and PEG + SNP groups were collected on the last day, and then immediately frozen in liquid nitrogen. Finally, they were then stored at $-80{ }^{\circ} \mathrm{C}$ for further use. Three replicates from each experiment were used for the analysis.

\subsection{Small RNA Library Construction and Sequencing}

Total RNA was isolated from alfalfa leaves of the CK, PEG, and PEG + SNP-treated groups using Trizol reagent (Invitrogen, Carlsbad, CA, USA) according to the instructions. The integrity of the RNA samples was tested using an Agilent Bioanalyzer 2100 system (Agilent Technologies, SantaClara, CA, USA) to ensure sequencing with qualified samples. After the sample was tested, the amount of $2.5 \mathrm{ng}$ was used as the starting amount of the RNA sample, the volume was supplemented to $6 \mu \mathrm{L}$ with water, and the library was constructed using the Small RNA Sample Pre Kit. T4 RNA ligase 1 and T4 RNA ligase 2 (truncated) were ligated to the 3' and 5' ends of the small RNA, respectively. The complementary DNA (cDNA) was synthesized by reverse transcription, amplified by PCR, and the target fragment was screened by gel separation technique. The 18-30 nt fragment was a small RNA library. The concentration of the library was tested using Qubit 2.0, the library concentration was diluted to $1 \mathrm{ng} / \mathrm{uL}$, and the insert size was detected using an Agilent 2100 bioanalyzer. The Q-PCR method was used to accurately quantify the effective concentration of the library to ensure library quality. After passing the library, the obtained RNA sequence was sequenced by Biomarket (Beijing, China) on the Illumina Hiseq 2500 platform, and the sequencing read length was single-end (SE) $50 \mathrm{nt}$. The raw data was saved in the National Center for Biotechnology Information (NCBI) sequence read file, and its Sequence Read Archive (SRA) data study deposit number is PRJNA551564.

\subsection{Bioinformatic Identification of Conserved and Novel miRNAs}

The original image data files obtained by sequencing were converted into Raw Reads by base call (Base Calling), and the results were stored in the FASTQ file format. High quality sequences (Clean Reads) were obtained by cleavage of the 3' linker sequence and removal of sequences shorter than 18 or longer than 30 nucleotides. Using Bowtie (version 1.0.0) software, clean reads were sequenced with the Silva database (http://www.arb-silva.de/), the GtRNAdb database (http://lowelab.ucsc.edu/GtRNAdb/), the Rfam database (http://rfam.xfam.org/) and the Repbase database (http://www.girinst.org/repbase/); non-coding RNA(ncRNA) including ribosomal RNA (rRNA), transport RNA (tRNA), small nuclear RNA (snRNA), small nucleolar RNA (snoRNA) and repeated sequences were filtered to obtain unannotated reads containing miRNA; and mapped reads were obtained by sequence comparison with the transcriptome of alfalfa. In comparison with the mature miRNA sequences in known miRBase (version 21). reads that were identical to known miRNAs were considered as identification of known miRNAs. Potential new miRNAs have been obtained by predicting the remaining readings by the online prediction software miRDeep2 (https://github.com/hangelwen/miR-PREFeR). 


\subsection{Differential Expression Analysis of Known and Novel miRNAs}

Differential expression analysis of mature miRNAs between CK and PEG samples, and CK and PEG + SNP samples was performed by DESeq (version 1.18.0) software. Differentially-expressed miRNAs were calculated using the expression of transcript per million (TPM) value, the expression of miRNA in treatment and control was statistically analyzed, and the expression was normalized by TPM algorithm. TPM values were calculated as follows: (actual miRNA count/total count of mapped reads) $\times 1,000,000$. Then, the fold-change and $p$-values were calculated from the normalized expression, and the $\log$ 2-ratio and scatter plots were then generated. A miRNA whose expression fold change satisfies $\mid \log 2$ (fold change) $\mid \geq 1$ and whose $p$-value $<0.05$ adjusted by the Benjamini-Hochberg method is considered as a differentially expressed miRNA.

\subsection{Prediction of miRNA Targets, Gene Ontology (GO), and KEGG Pathway Analysis}

The plant miRNA target gene prediction software TargetFinder (version 1.6) was used to predict the target genes of differentially expressed miRNA. Mature miRNA sequences were used as queries to search for potential target mRNAs in the Medicago sativa transcriptome, and default parameters were used for target prediction. By using BLAST (version 2.2.26) sequence alignment software, the predicted target gene sequence was compared with the database for NCBI non-redundant protein sequences (NR) (ftp://ftp.ncbi.nih.gov/blast/db/), swiss-prot (http://www.uniprot.org/), GO (http: //www.geneontology.org/), clusters of orthologous groups (COG) (http://www.ncbi.nlm.nih.gov/COG/), KEGG (http://www.genome.jp/kegg/), protein homologous cluster (KOG) (http://www.ncbi.nlm.nih. gov/KOG/), and homologous protein family (Pfam) (http://pfam.xfam.org/) to obtain the annotation information of the target gene.

\subsection{Validation of miRNA and Target Gene Expression with qRT-PCR}

The expression of some miRNAs and target genes were validated by quantitative real-time PCR (qRT-PCR). qRT-PCR was performed on Analytikjena -qTOWER2.2 fluorescence quantitative PCR instrument (Germany). Similar RNA samples were used with Illumina sequencing in qRT-PCR analysis. Reverse transcriptase reactions contained DNase-treated total RNA and a gene-specific stem-loop real-time (RT) PCR primer. According to the instructions, reverse transcription was performed using a TUREscript 1st Stand cDNA SYNTHESIS (Aidlab biotechnologies CO. Ltd, Beijing, China) kit using a $20 \mu \mathrm{L}$ reaction system: total RNA $1000 \mathrm{ng}, 4 \mu \mathrm{L} 5 \times$ RT Reaction Mix, $0.8 \mu \mathrm{L}$ Rondam primer/oligodT, $0.8 \mu \mathrm{L}$ TUREscript H- RTase/RI Mix, and finally adding $\mathrm{RNase}^{-F r e e ~} \mathrm{dH}_{2} \mathrm{O}$ to $20 \mu \mathrm{L}$. Reverse transcription was performed at $42{ }^{\circ} \mathrm{C}$ for $40 \mathrm{~min}$, then reverse transcription at $65{ }^{\circ} \mathrm{C}$ for $10 \mathrm{~min}$ to obtain cDNA. The following temperature program was used to perform the RT reaction for $3 \mathrm{~min}$ at $95^{\circ} \mathrm{C}, 40$ cycles of $10 \mathrm{~s}$ at $95^{\circ} \mathrm{C}, 30 \mathrm{~s}$ at $58^{\circ} \mathrm{C}$, and then the holding time was $4 \mathrm{~s}$ at $65^{\circ} \mathrm{C}$. All reactions were repeated three times. The mtr-miR7701-5p and c115758.graph_c0 were served as miRNA and mRNA for internal control, respectively. The relative expression of the target gene in each sample was calculated automatically by the instrument software qPCRsoft3.2, and manually calculated. The relative expression level of miRNAs and the predicted target genes was calculated using the $2^{-\Delta \Delta C T}$ method, and relative expressions were shown as $\log _{2}$ fold change. The miRNAs and target genes primers are listed in Table S1.

\section{Results}

\subsection{Deep Sequencing Results of Alfalfa Small RNA (sRNA) Libraries}

A total of 9 sRNA libraries (one library with weak correlation in CK and PEG + SNP treatment groups was deleted to ensure the accuracy of the subsequent analysis) comprising three samples (CK: leaves with normal water, PEG: leaves with drought stress, PEG + SNP: leaves with drought stress and SNP treatment) were generated using the Illumina HiSeq 2500 platform, and the sequencing data of the three samples were averaged. By extracting both the adapter and low amount of reads, 
the three samples obtained 23,528,741 (accounting for 87.63\% of raw reads), 18,046,004 (accounting for $74.41 \%$ of raw reads), and $15,076,692$ (accounting for $73.49 \%$ of raw reads) clean reads. High-quality sRNA reads were mapped to the alfalfa transcriptome sequence using Bowtie software. The CK, PEG, and PEG + SNP libraries were matched to 10,366,134, 7,651,367, and 6,487,853 mapped raw. In order to classify the sRNA sequenced reads into different categories and identify all the miRNA sequences in the libraries, the researchers mapped the reads to specific databases with Bowtie. At all levels, the tRNA, miRNA, snoRNA, and rRNA reads were annotated as the clean sRNA in these libraries. The reads of 1,267,908, 1,180,088, and 871,935 miRNAs were mapped to the CK, PEG, and PEG + SNP groups, respectively. Therefore, the percentage obtained for miRNA reads in PEG were more abundant than CK and PEG + SNP groups. Table 1 shows the details of raw reads, clean reads, and sRNA sequencing in three samples.

Table 1. Alfalfa small RNA (sRNA) sequencing datasets. Statistics of sRNA sequences for CK (normal water), PEG (polyethylene glycol-6000) drought stress, and PEG + SNP (sodium nitroprusside; drought stress and SNP treatment) libraries from Medicago sativa L. "Sandeli". Percentage refers to the net read code, which is equal to the ratio of each category to raw reads. rRNA: filtering ribosomal RNA, snoRNA: nucleolar small RNA, tRNA: transport RNA.

\begin{tabular}{ccccccc}
\hline \multirow{2}{*}{ Category } & \multicolumn{2}{c}{ CK } & \multicolumn{2}{c}{ PEG } & \multicolumn{2}{c}{ PEG + SNP } \\
\cline { 2 - 6 } & Count & Percentage & Count & Percentage & Count & Percentage \\
\hline Raw reads & $26,849,326$ & $100.00 \%$ & $24,252,135$ & $100.00 \%$ & $20,514,941$ & $100.00 \%$ \\
Clean reads & $23,528,741$ & $87.63 \%$ & $18,046,004$ & $74.41 \%$ & $15,076,692$ & $73.49 \%$ \\
Mapped to genome & $10,366,134$ & $38.61 \%$ & $7,651,367$ & $31.55 \%$ & $6,487,853$ & $31.63 \%$ \\
miRNAs & $1,267,908$ & $4.72 \%$ & $1,180,088$ & $4.87 \%$ & 871,935 & $4.25 \%$ \\
rRNA & $2,931,163$ & $10.92 \%$ & $3,527,200$ & $14.54 \%$ & $1,974,535$ & $9.62 \%$ \\
snoRNA & 3746 & $0.01 \%$ & 7001 & $0.03 \%$ & 10,497 & $0.05 \%$ \\
tRNA & 234,634 & $0.87 \%$ & 333,859 & $1.38 \%$ & 417,378 & $2.03 \%$ \\
Without annotation & $20,273,991$ & $75.51 \%$ & $14,113,193$ & $58.19 \%$ & $12,602,624$ & $61.43 \%$ \\
\hline
\end{tabular}

sRNA of different lengths have different functions. For example, 21/22 nt sRNAs are involved in post-transcriptional gene silencing and mRNA cleavage, whereas $24 \mathrm{nt}$ sRNAs are primarily responsible for transcriptional gene silencing and DNA methylation. As seen in the distribution of sRNA length, 21 to $24 \mathrm{nt}$ accounted for the majority, and the $24 \mathrm{nt}$ category was the most abundant compared to other lengths, followed by $21 \mathrm{nt}$ and $22 \mathrm{nt}$ (Figure 1). As shown in Figure 1, the lengths of sRNA from CK, PEG, and PEG + SNP showed similar rules, but some slight differences still exist. The order of 24 nt sequences in each sample was found to be CK > PEG + SNP > PEG, and the 21-22 nt sequence exhibited an opposite expression profile compared to $24 \mathrm{nt}$. These consequences indicated increased levels. When alfalfa was subjected to drought stress, 21, 22, and $24 \mathrm{nt}$ miRNAs reduced their role in post-transcriptional gene silencing, whereas exogenous nitric oxide can raise 24 nt sRNA-mediated transcriptional gene silencing, causing RNA-directed DNA methylation to occur.

\subsection{Conserved miRNAs from Alfalfa}

By comparing the known plant miRNAs in miRBase, the study identified some conserved miRNAs obtained by high-throughput sequencing. A total of 90 unique mature miRNA sequences from 46 families were found in alfalfa. We found 88, 86, and 82 miRNAs from three treatment groups, and they belonged to 44 miRNA families. The miR530 family specifically expressed in the CK and PEG + SNP were guided by the miR2632 family, as shown in Table S2. In comparison with other miRNA family readings, miR2109 and miR159 showed the highest level (Table S3). The miR156 family contained 8 miRNAs with the most members. The abundance of conserved miRNAs of the top 10 most highly expressed in all libraries is shown in Figure 2. 


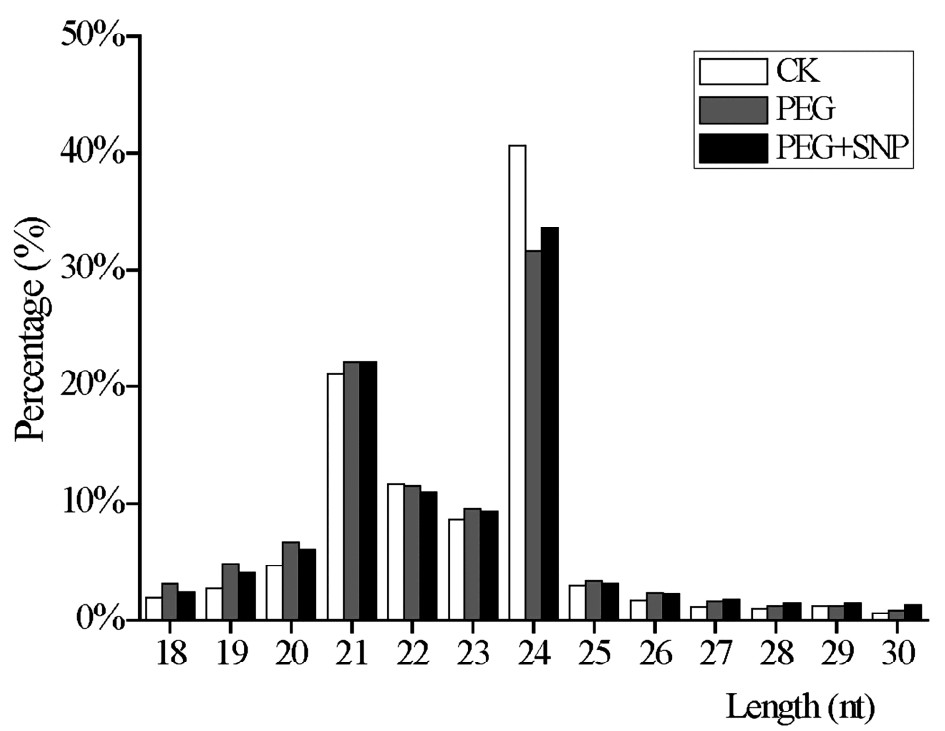

Figure 1. The length distribution of sRNAs in the libraries of CK, PEG, and PEG + SNP in alfalfa.

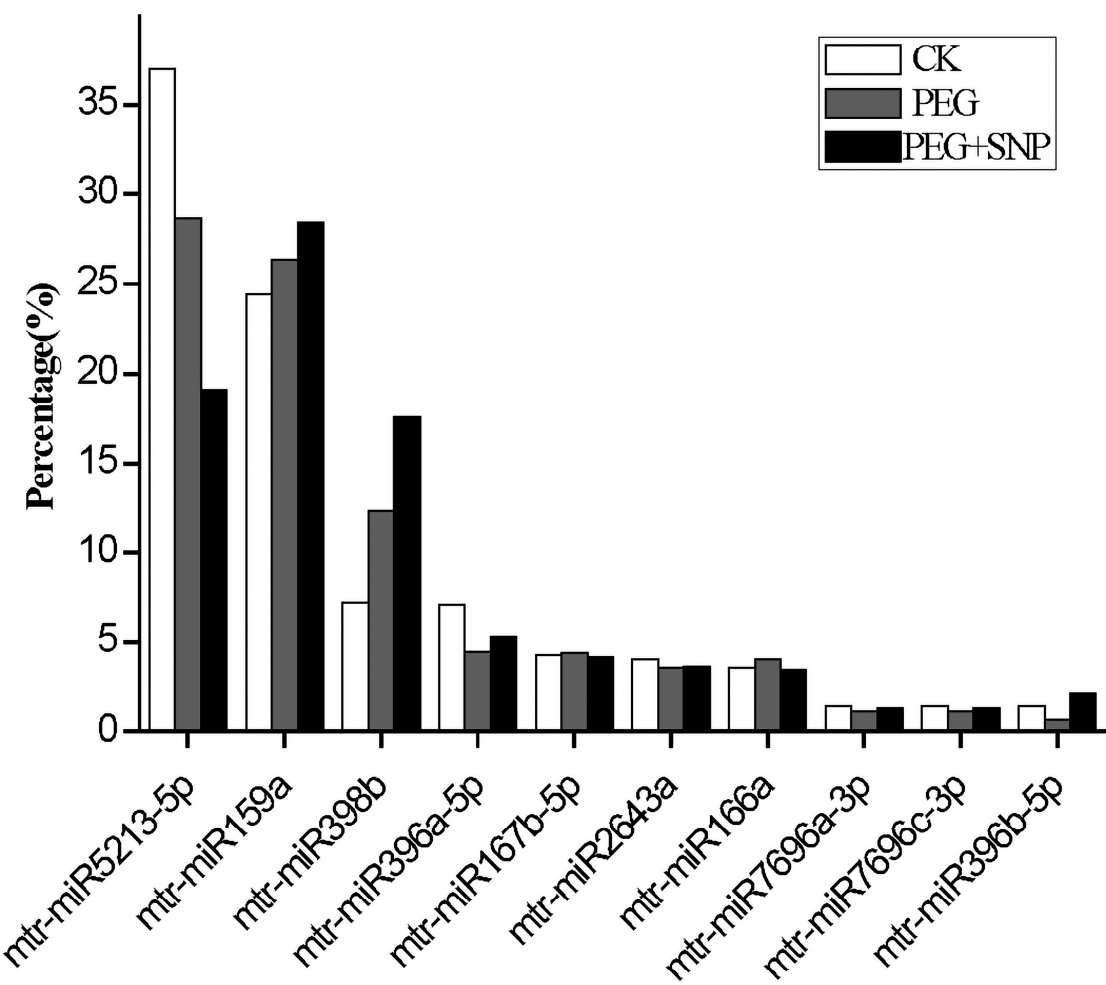

Figure 2. Abundance of the top 10 highly-expressed conserved miRNAs in the seven alfalfa libraries.

\subsection{Check of Unknown miRNAs in Medicago sativa L. "Sandeli"}

To evaluate unknown miRNAs in the clean reads, miRDeep2 was used to forecast putative new miRNAs after removing the verified unknown miRNAs. The total of 177 new miRNAs were discovered. In the library of CK (169), PEG (172), and PEG + SNP (154), novel miRNAs were detected as well. Detailed information about the unknown miRNA sequences, extent of reads, pre-miRNA sequences, genome ID, miRNA length, and Fragments Per Kilobase Million (FPKM) are listed in Table S4. Most new miRNAs have lower readings when compared to conserved miRNAs, and only 9 novel miRNAs had more than 1000 reads (Table S5). Table 2 shows information of 10 new miRNAs, including length, score total, expressional reads, guanine $(G)+$ cytosine $(C)$ contents, and hairpin energy, with high confidence found in all libraries. 
Table 2. Ten novel miRNAs identified from Medicago sativa L. transcriptional databases.

\begin{tabular}{|c|c|c|c|c|c|c|}
\hline Name & Sequence & Score_Total & Expressional Reads & $G+C$ Contents & Hairpin Energy & Length \\
\hline c104339.graph_c0_3174 & UUUCAUUCCAUAUGUCAUCUAG & $17,723.5$ & 14,392 & $31.82 \%$ & -53.6 & 22 \\
\hline c111080.graph_c1_10087 & GGCGGAUGUAGCCAAGUGGA & $1,721,964.4$ & 730,326 & $60.00 \%$ & -53.2 & 20 \\
\hline c114875.graph_c0_14536 & UUCAUUUCUAAAAUAGGCAUUG & 9543.7 & 7780 & $27.27 \%$ & -59.2 & 22 \\
\hline c116131.graph_c0_16318 & CGGGAUCGGAGAUUAGAGAAU & $146,625.1$ & 106,408 & $47.62 \%$ & -73.0 & 21 \\
\hline c86020.graph_c0_42677 & UACGUCUCUGUCUUUCGGGUUG & $16,890.1$ & 13,750 & $50.00 \%$ & -74.6 & 22 \\
\hline c99918.graph_c0_48282 & UUAAUCAAGGAAAUCACAGUC & $13,503.9$ & 13,046 & $33.33 \%$ & -55.0 & 21 \\
\hline c102504.graph_c0_1594 & AUGGUUCUUGUUCAGUAGAGU & $30,812.8$ & 6815 & $38.10 \%$ & -78.4 & 21 \\
\hline c91116.graph_c0_44142 & UUGUGGAACAUAGAAGCACGUG & 9771.1 & 2317 & $45.45 \%$ & -63.6 & 22 \\
\hline c121624.graph_c0_25405 & UUGAUUCUCAUCACAACUUGG & $14,278.7$ & 7926 & $38.10 \%$ & -66.2 & 21 \\
\hline c111105.graph_c0_10123 & UUUGGCAUUCUGUCCACCUCC & 8328.7 & 7532 & $52.38 \%$ & -55.6 & 21 \\
\hline
\end{tabular}




\subsection{The Response of miRNA to Drought Stress and Exogenous Nitric Oxide under Drought Stress}

To identify miRNAs with response to drought and exogenous NO response under drought stress, the study compared the expressions of known and new miRNAs in CK and PEG, and CK and PEG + SNP samples. The researchers detected 32 miRNAs that responded to drought treatment (including 14 known miRNAs and 18 newly predicted miRNAs) and 55 miRNAs that responded to exogenous NO under drought stress (including 24 known, 31 unknown). Under PEG treatment, 11 of the 14 known miRNAs were up-regulated and 3 were down-regulated (Figure 3a, Table S6); from the total of 18 differentially expressed new miRNAs, 8 were up-regulated and 10 were down-regulated. Under the treatment of PEG + SNP, 14 of the 24 known miRNAs were up-regulated and 10 were down-regulated (Figure 3b, Table S7); 14 of the 31 new miRNAs were up-regulated and 17 were down-regulated. Detailed information of the drought-responsive and exogenous nitric oxide-responsive responses under drought stress miRNAs, such as FPKM, log fold-change, and $p$-value, are shown in Tables S6 and S7, respectively.

From the Tables S6 and S7, a huge number of miRNAs were not only silenced, but were induced by the NO under drought stress. The researchers identified 10 conserved miRNAs that were only regulated by exogenous NO under drought stress (Table 3). Four of them were silenced, including mtr-miR398a-5p, mtr-miR5232, mtr-miR5295a, and mtr-miR5559-5p. In addition, mtr-miR156a, mtr-miR399a, mtr-miR399c, mtr-miR399q, mtr-miR5213-5p, mtr-miR5752a, and mtr-miR7696a-5p were induced. More members of the miR399 family were regulated by exogenous NO. It must be mentioned that the expression of the homologous miRNAs, belonging to the same miRNA family, was consistently similar under drought stress. For instance, the miR156 family was all up-regulated, whereas the miR399 family was all down-regulated.

(a)

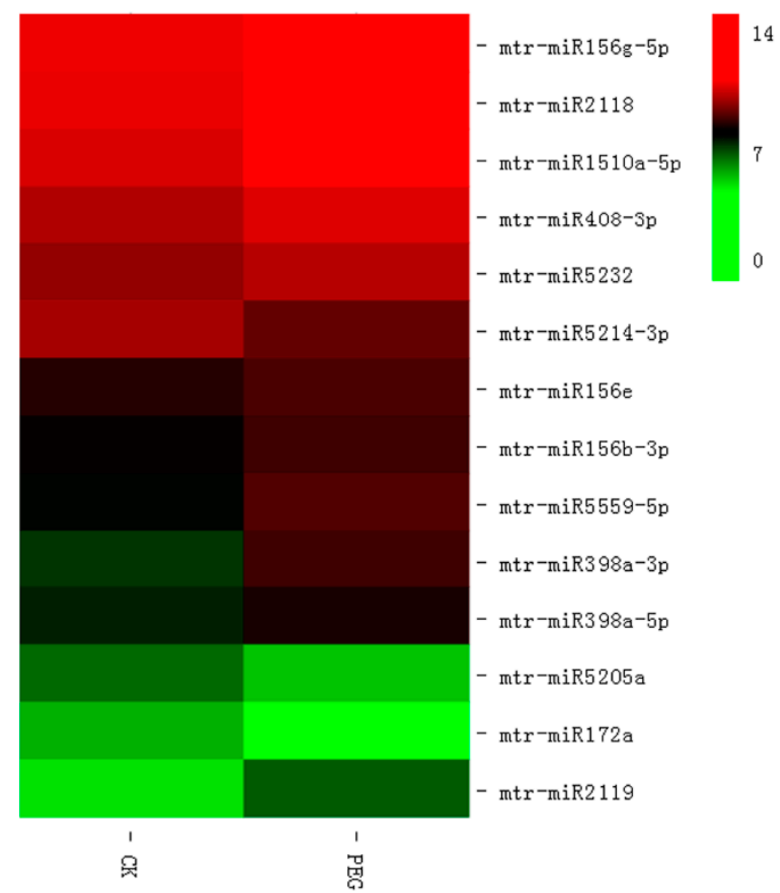

Figure 3. Cont. 
(b)

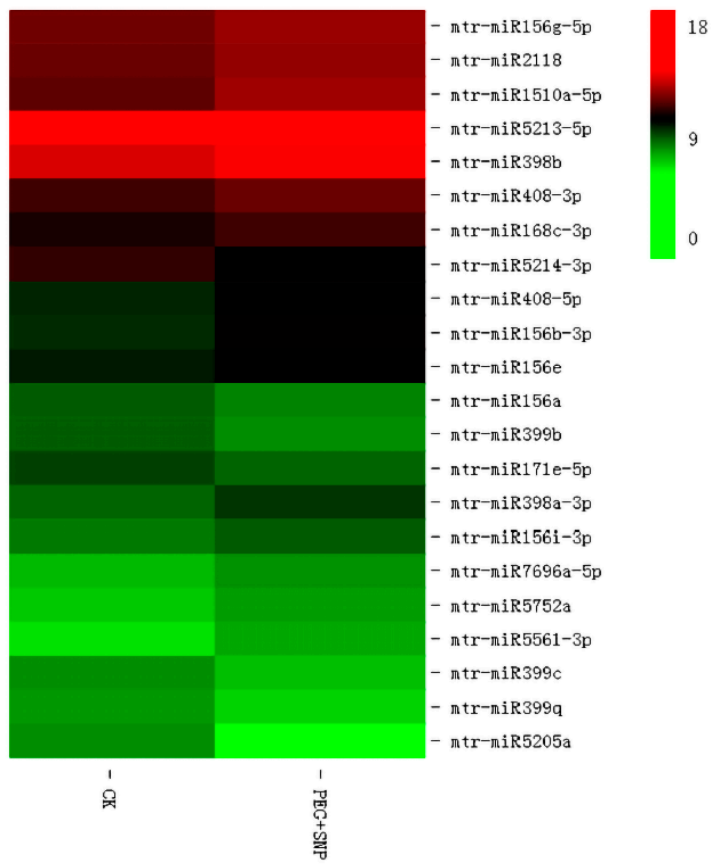

Figure 3. Differentially-expressed conserved miRNAs upon different treatment in alfalfa. (a) Conserved miRNAs from CK and PEG samples. (b) Conserved miRNAs from CK and PEG + SNP samples. The upregulated miRNAs are showed in red, whereas downregulated miRNAs are shown in green.

In addition, Figure 4a shows that 10 differentially-expressed conservative miRNAs were shared by PEG and PEG + SNP, but their FPKM were different. This indicated that the miRNAs were prerequisite in responding to drought stress, such as mtr-miR1510a-5p, mtr-miR172a, and mtr-miR408-3p. Figure $4 b$ shows that common 14 miRNAs (7 raised and 7 cutted) were discovered in PEG and PEG + SNP samples. Novel miRNAs in PEG + SNP were more than those in PEG. This indicated that NO caused the emergence of more novel miRNAs. Overall, these new miRNAs may aid in clarifying the drought-responsive and exogenous nitric oxide-regulative mechanism under drought stress.

a

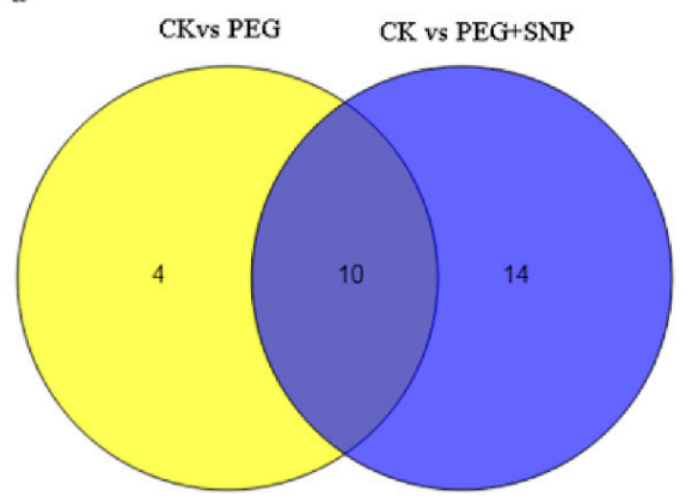

b

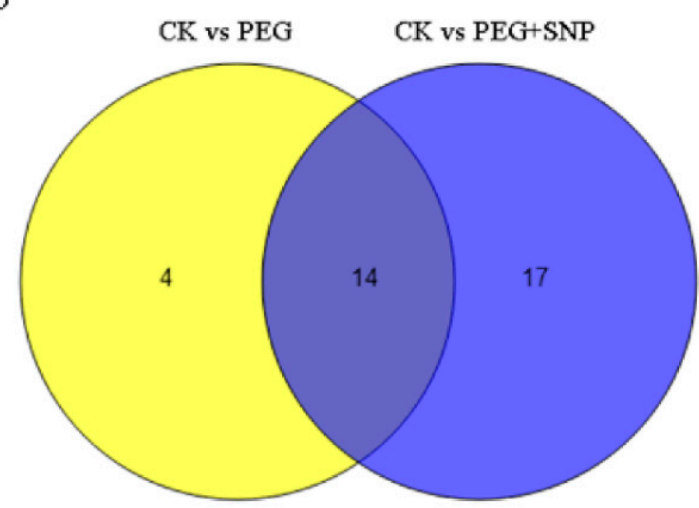

Figure 4. Diagrams of alfalfa miRNAs identified in the three samples: (a) conserved miRNAs, (b) novel miRNAs. 
Table 3. Ten differentially expressed conserved miRNAs that can specifically induced or silenced by exogenous nitric oxide, including the miRNA name, sequences, target gene or protein, and description of function.

\begin{tabular}{|c|c|c|c|c|}
\hline miRNAs & Sequences & Target Gene or Protein & Description of Function & Reference \\
\hline mtr-miR156a & tgacagaagagagagagcaca & SQUAMOSA promoter-binding-like protein (SPL) genes. & $\begin{array}{l}\text { Anthocyanin biosynthesis; } \\
\text { vegetative phase transition. }\end{array}$ & {$[30,36,37]$} \\
\hline $\begin{array}{l}\text { mtr-miR399a } \\
\text { mtr-miR399c } \\
\text { mtr-miR399q }\end{array}$ & $\begin{array}{l}\text { tgccaaaggagatttgcccag } \\
\text { tgccaaaggagatttg } \\
\text { ccctgtgccaaaggagagctgctctt }\end{array}$ & $\begin{array}{l}\text { Phosphate transporter; high affinity inorganic phosphate } \\
\text { transporter. }\end{array}$ & Pi uptake. & {$[38,39]$} \\
\hline mtr-miR5213-5p & tacgtgtgtcttcacctctgaa & Disease resistance protein. & Response to salt/alkali stress. & {$[40,41]$} \\
\hline mtr-miR5752a & cattgtttggtttagtacaaa & $\begin{array}{l}\text { Starch synthase; } \\
\text { amino acid binding; } \\
\text { metal ion binding. }\end{array}$ & $\begin{array}{l}\text { amylopectin biosynthetic; } \\
\text { response to hypoxia; } \\
\text { regulation of ethylene-activated; } \\
\text { metabolic process; } \\
\text { metal ion transport. }\end{array}$ & [42] \\
\hline mtr-miR7696a-5p & tcaagttctcataattcaaaa & $\begin{array}{l}\text { Chitin binding; } \\
\text { protein kinase. }\end{array}$ & $\begin{array}{l}\text { Innate immune response; } \\
\text { protein phosphorylation; } \\
\text { cell wall macromolecule catabolic. }\end{array}$ & [43] \\
\hline mtr-miR398a-5p & ggagtgacactgagaacacaag & $\begin{array}{l}\mathrm{Cu} / \mathrm{Zn} \text {-superoxide dismutase copper chaperone. } \\
\text { Glycoside hydrolase; }\end{array}$ & Defense against reactive oxygen toxicity. & [44] \\
\hline mtr-miR5232 & tacatgtcgctctcacctgaa & $\begin{array}{l}\text { type IIB calcium ATPase } \\
\text { protein kinase. }\end{array}$ & Participate in metabolism. & [41] \\
\hline mtr-miR5559-5p & tacttggtgaattgttggatc & $\begin{array}{l}\text { inorganic diphosphatase } \\
\text { magnesium ion binding. }\end{array}$ & $\begin{array}{l}\text { Response to cadmium ion; } \\
\text { phosphate-containing compound metabolic. }\end{array}$ & [29] \\
\hline
\end{tabular}


3.5. Prediction and Functional Classification of Target Genes of Drought Stress Response Type and Exogenous Nitric Oxide Reactive miRNA under Drought Stress

The current study used the TargetFinder server to predict the target sequence of differentially expressed miRNAs. A total of 86 and 157 putative targets were retrieved for differentially-expressed miRNAs in PEG and PEG + SNP, respectively. Tables S8 and S9 show miRNA and corresponding target genes. The results reported that one miRNA can act on multiple mRNAs, and different miRNAs can target the same target gene (Tables S8 and S9). Due to lack of genome information, some miRNA targets were not predicted.

For example, Table S10 shows that squamosa promoter-binding-like protein (SBP) transcription factors were targeted by mtr-miR156a $\backslash$ e $\backslash g-5 p$ in the PEG + SNP sample, and was involved during plant growth, morphogenesis, stress response, floral organ development, and plant photoreaction. However, only mtr-miR156e $\backslash g-5 p$ regulates SBP transcription factors under drought stress $[45,46]$. This indicates that NO can induce more miRNA and its target genes to participate in drought resistance. In addition, exogenous NO specifically regulated myb-like DNA-binding domain (MYB) transcription factors under drought stress, which has shown various cellular processes involved in regulation including cell cycle, cell morphogenesis, and biotic and abiotic stress responses $[47,48]$. In particular, MYB transcription factors play an important role in phenylpropanoid metabolism in plants [49]. The results predicted that target genes are also involved in alfalfa phenylpropanoid metabolism.

To further comprehend the potential effect of drought-responsive and exogenous nitric oxide-regulative miRNAs in alfalfa, GO and KEGG pathway analyses were carried out on all target genes. The results indicated that 59 genes have been annotated and $114 \mathrm{GO}$ terms were enriched under drought stress (Tables S11 and S12), including 68 biological action processes, 33 molecular functions, and 13 cell compositions. Most enriched GO terms were related to plant stress tolerance, for instance, defense response (GO:0006952), response to water deprivation (GO:0009414), respiratory burst involved in defense response (GO:0002679), and carbohydrate transport (GO:0008643). GO terms related to hormone metabolism were also enriched, including response to brassinosteroid (GO:0009741), abscisic acid-activated signaling pathway (GO:0009738), ethylene-activated signaling pathway (GO:0009873), and ethylene biosynthetic process (GO:0009693).

Many GO terms were also differently enriched in PEG + SNP samples. A total of 114 genes were annotated and $214 \mathrm{GO}$ terms were regulated by extraneous nitric oxide under drought stress. Also, 117 biological reaction processes, 70 molecular functions, and 27 cell compositions were enriched (Tables S12 and S13). From Figure 5, in PEG + SNP samples, the number of biological processes, molecular functions, and cellular components increased by 69.57\%, 1.33 and 1.08 times more than PEG samples, respectively, and exogenous NO regulated the initiation of more genes and multiple biological processes to respond to drought stress. Participation of more cellular components will aid alfalfa in improving the efficiency of drought response. As compared with drought stress, exogenous NO regulated carbohydrate metabolism in particular, which included the cellulose biosynthetic process (GO:0030244), starch biosynthetic process (GO:0019252), galactolipid biosynthetic process (GO:0019375), amylopectin biosynthetic process (GO:0010021), and carbohydrate transmembrane transport (GO:0034219).

A total of 6 and 12 KEGG pathways for the miRNA target gene were enriched in PEG and PEG + SNP samples (Table S14), respectively. It must be noted that oxidative phosphorylation, phenylpropane metabolism, ubiquinone and other terpenoid metabolism, alpha-linolenic acid metabolism, and RNA transport were all enriched in both samples. Endocytosis was specifically enriched only in PEG samples. In PEG + SNP samples, exogenous NO specifically regulated metabolic pathways such as ascorbic acid, tyrosine metabolism, starch and sucrose metabolism, amino acid synthesis, ribosome, and protein transport in response to drought stress. 


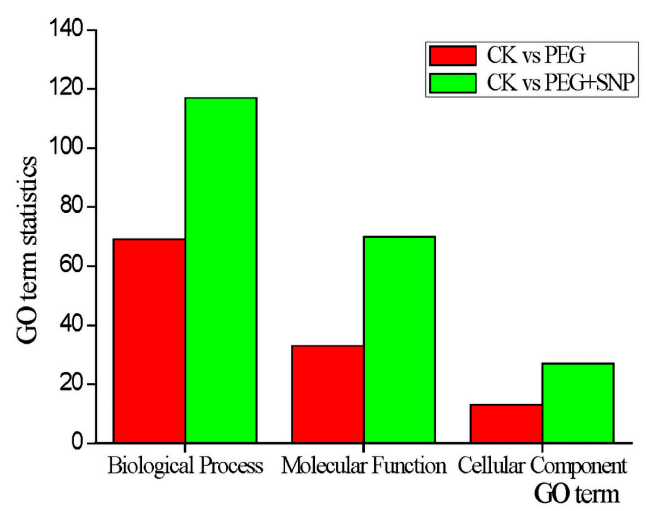

Figure 5. The number of gene ontology (GO) terms in different comparisons.

\subsection{Validation of the Expression of miRNAs and Their Targets}

In order to verify the expression of miRNA and its target genes, 9 miRNAs (mtr-miR5214-3p, mtr-miR2199, mtr-miR396a-5p, mtr-miR399b, mtr-miR408-5p, mtr-miR5752a, unconservative_c105676.graph_c0_4408, unconservative_c103008.graph_c0_2076, and unconservative_c103018.graph_c0_2090) and their target sequences were chosen for qRT-PCR validation (Figure 6). Except for mtr-miR5214-3p, the other qRT-PCR results of miRNA profiles were similar to the sequencing results. The target gene was expressed in the opposite way. For example, deep sequencing results exhibited that mtr-miR2199 was down-regulated and their corresponding target gene c114003.graph_c0 showed raising patterns. mtr-miR408-5p and mtr-miR5752a were significantly up-regulated, and for the corresponding target gene of interest c115697.graph_c0 and c117794.graph_c0 showed decreasing patterns. The results of qRT-PCR confirmed that the RNA sequencing results were authentic.

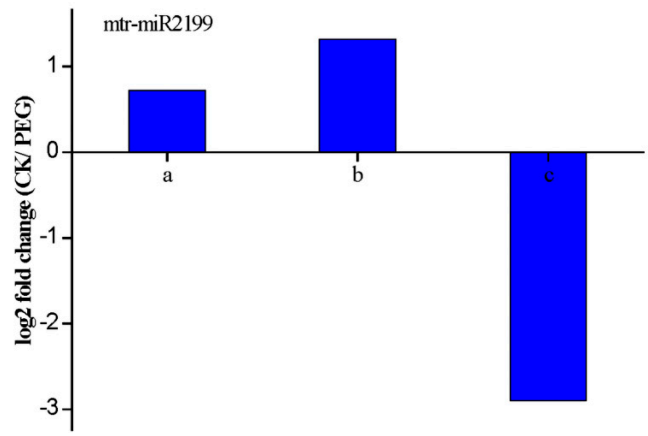

(a) qRT-PCR validation of mtr-miR2199 and target genes in alfalfa

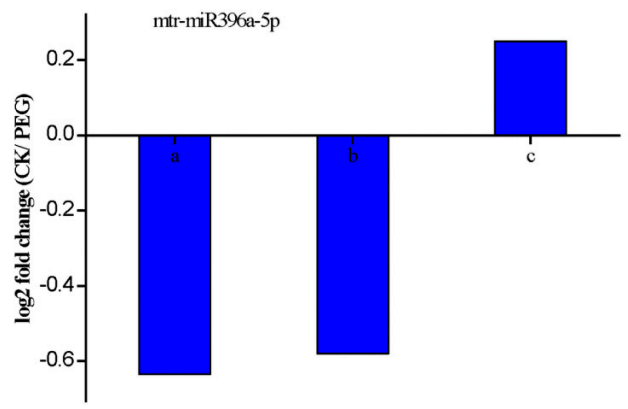

(b) qRT-PCR validation of mtr-miR396a-5p and target genes in alfalfa

Figure 6. Cont. 


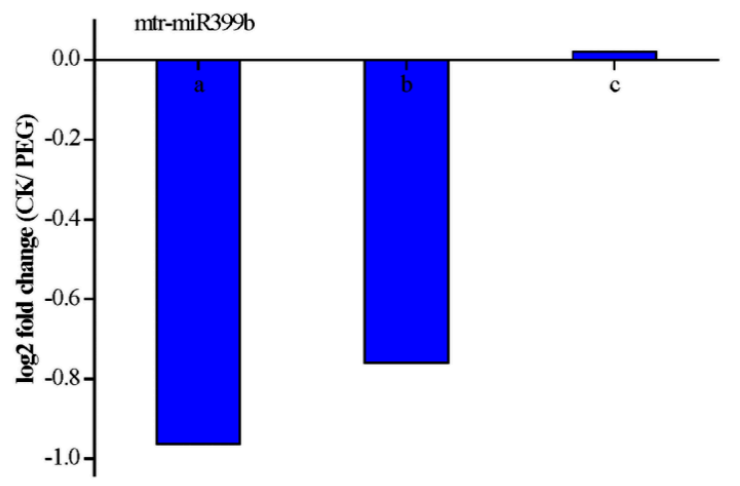

(c) qRT-PCR validation of mtr-miR399b and target genes in alfalfa

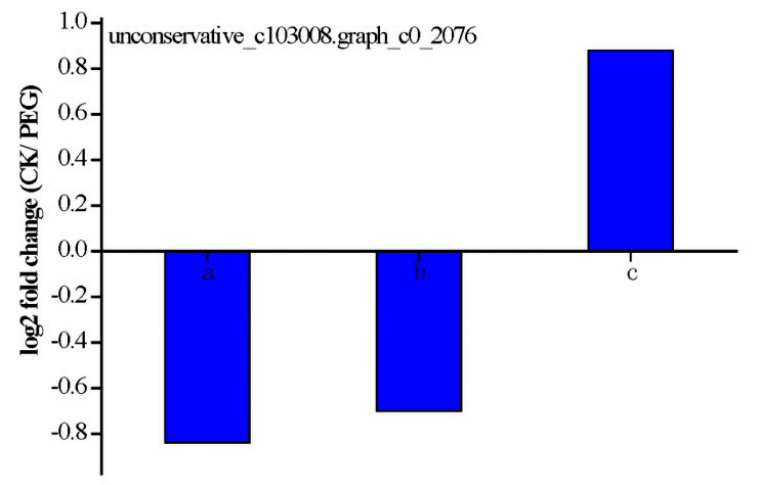

(d) qRT-PCR validation of unconservative_c103008.graph_c0_2076 and target genes in alfalfa

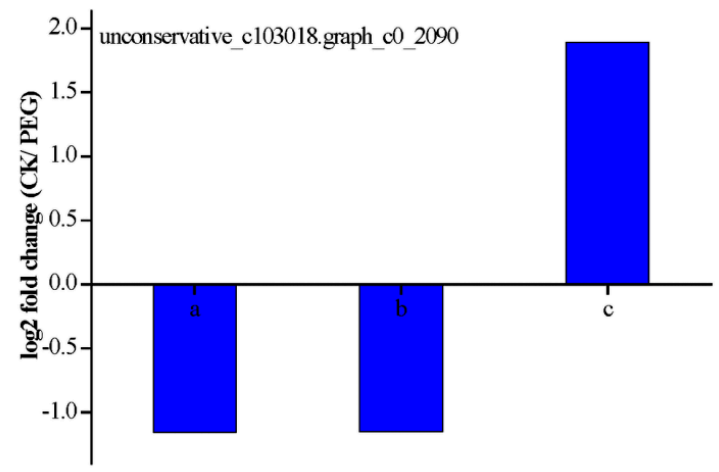

(e) qRT-PCR validation of unconservative_c103018.graph_c0_2090 and target genes in alfalfa

Figure 6. Cont. 


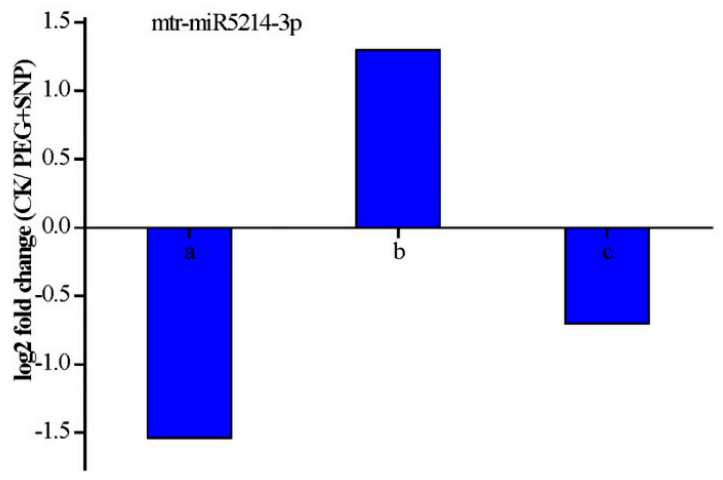

(f) qRT-PCR validation of mtr-miR5214-3p and target genes in alfalfa

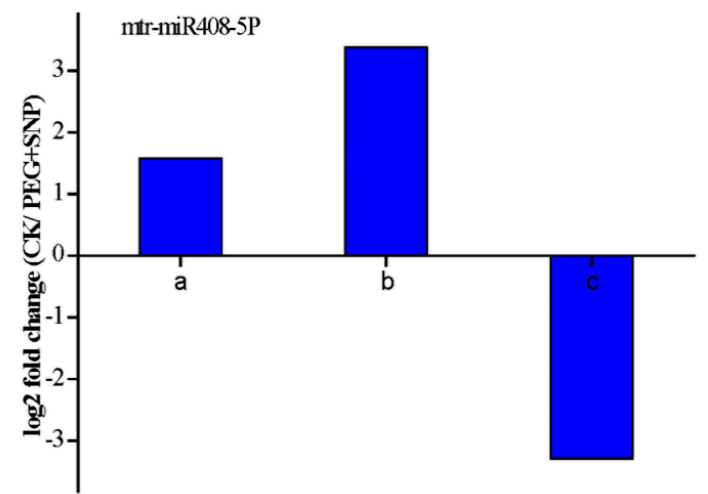

(g) qRT-PCR validation of mtr-miR408-5p and target genes in alfalfa

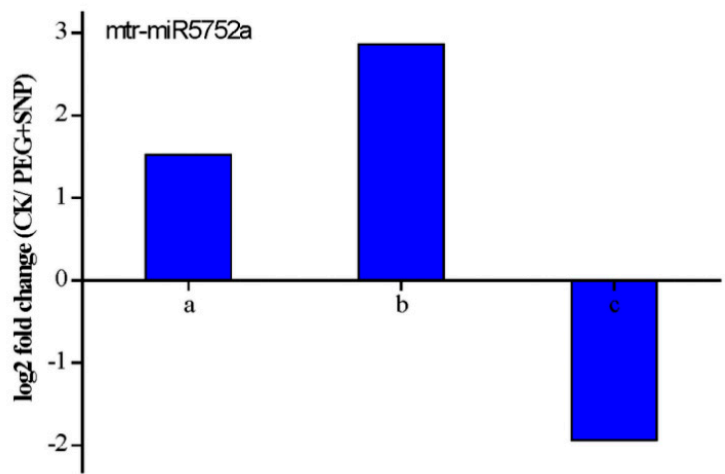

(h) qRT-PCR validation of mtr-miR5752a and target genes in alfalfa

Figure 6. Cont. 


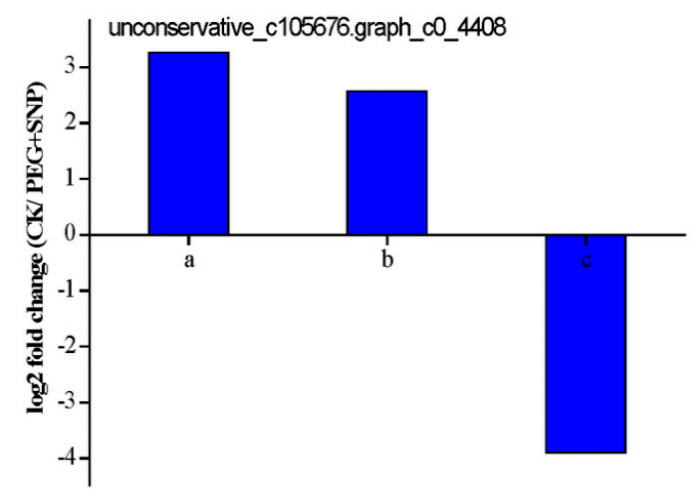

(i) qRT-PCR validation of unconservative_c105676.graph_c0_4408 and target genes in alfalfa

Figure 6. qRT-PCR validation of nine miRNAs and target genes in alfalfa. ( $a$ in the $x$-coordinate) miRNA expression patterns from deep sequencing, ( $b$ in the $x$-coordinate) miRNA expression patterns from qRT-PCR, (c in the $\mathrm{x}$-coordinate) target genes expression patterns.

\section{Discussion}

Drought stress is one of the environmental stresses hindering the growth and development of plants [50]. There are three types of interactive modification that respond to drought stress in plants: the alteration in gene expression (over, under, and co-expression), dynamics in protein metabolism, and alterations in the metabolic pool [51,52]. The three types of modification above may all confer plant resistance or tolerance to drought stress. Studies have confirmed that miRNA can regulate gene expression, protein metabolism, and metabolic pool. In addition, sequencing techniques use for small RNAs improve the abundance of miRNA species. Many researchers have begun to utilize miRNA to reveal the drought-resistance mechanism of plants, such as Arabidopsis and Oryza sativa $[38,53]$. Functioning as second messenger of plants, NO release SNP agent, which has been shown to improve plant stress resistance. However, the report for miRNAs did reveal whether the molecular mechanism of exogenous $\mathrm{NO}$ can improve alfalfa drought resistance.

The current study showed that nine libraries were constructed from alfalfa treated with normal watering, drought stress, and exogenous NO under drought stress, and also that two uncorrelated libraries were discarded. All the libraries were sequenced on the Illumina Hiseq 2500 platform. The researchers identified that the known and new miRNAs induced by exogenous NO were under drought stress and drought correlation, and their predicted target genes were classified accordingly. According to the sequence, approximately 15-23 million clean reads for each library were generated. The reads for drought stress samples were decreased and the control, known miRNAs reads containing the same tendency-indicated that the drought treatment restrained expression of some miRNAs. Therefore, more miRNA-related genes and pathways responded to drought stress, which was similar to several previous studies on Medicago sativa L. cv. Aohan and tomato [26,34]. Coincidentally, exogenous NO enhanced this trend to decrease the harming of drought stress.

The results reported that 88,86 , and 82 miRNAs were obtained from the CK, PEG, and PEG + SNP groups in alfalfa, respectively. Among them, the miR156 family reported the highest members. The study also found that mtr-miR5213-5p, mtr-miR159a, mtr-miR398b, mtr-miR396a-5p, mtr-miR167b-5p, mtr-miR2643a, mtr-miR166a, mtr-miR7696a-3p, mtr-miR7696c-3p, and mtr-miR396b-5p were the most enriched miRNAs in response to drought stress. These highly expressed miRNAs may regulate gene expression. It is worth mentioning that mtr-miR5213-5p were the highly expressed miRNAs-this is consistent with Li et al.'s study in alfalfa under natural drought stress [30]. Previous studies have shown that mtr-miR5213-5p also regulated gene expression of alfalfa in response to salt-alkali stress [40]. These indicate that the expression of mtr-miR5213-5p is important in responding to drought and saline-alkali stresses. 
Furthermore, the researchers predicted 177 new miRNAs in all libraries, and the expression levels of most new miRNAs were lower, which is consistent with previous reports [54,55]. Previous results are limited in terms of analyzing the differential expression of new miRNAs between different libraries. The possible reason is that novel miRNAs can only be induced to express under certain conditions such as environmental factor induction, and it is suggested that further investigation is needed to explore the expression mechanism.

The researchers compared the expression profiles of mature miRNAs in PEG and PEG + SNP samples with the control group (CK) to identify differentially expressed miRNAs. Differing from the expression of miRNAs under natural drought, the present results reported that 14 known miRNAs in the PEG sample and 24 known miRNAs in the PEG + SNP sample were considered as drought-responsive and nitric oxide-responsive under drought stress miRNAs [30]. The results also reported that two known miRNAs were identified by PEG-simulated drought treatment as compared to natural drought. This may have been due to the different manners of drought treatment. In addition, it was confirmed that miR408 and miR2118 in alfalfa leaves are associated with drought, and specifically identified in mtr-miR172a, mtr-miR398a-3p, mtr-miR5295a, and mtr-miR398a-5p, which are the drought-responsive miRNAs. Similar results were reported for the tomatoes, Lycopersicon esculentum, Medicago sativa, and Medicago truncatula [32,56]. Possible causes leading to these different consequences are (a) different alfalfa varieties, (b) different treatment conditions (natural drought versus PEG treatment), (c) different alfalfa developmental stages, (d) different growth environment, and (e) possible false positives in sequencing. In addition, miR1510 and miR5559 are specifically found in leguminous plants [30], as the current results showed that mtr-miR1510a-5p and mtr-miR5559-5p were identified and differentially expressed under drought stress. The above results are conducted at the level of $\log _{2}$ fold change $>1$, $p$-value $<0.05$.

Exogenous NO can induce mtr-miR156a, mtr-miR399a, mtr-miR399c, mtr-miR399q, and mtr-miR5213-5p down-regulated expression in alfalfa under drought stress. Past studies showed that the inhibition of gene translation or degradation of targeted mRNA can achieve miRNA regulation of gene expression [20]. It was predicted that the miRNA targets whose exogenous NO is down-regulated may play a positive regulatory role in drought stress response. mi156a are important miRNA for plant for abiotic stress responses, growth, and development [57]. miR156 can regulate plant stress tolerance by modulating SQUAMOSA promoter-binding-like protein (SPL) genes that function in anthocyanin biosynthesis [36]. We thought exogenous NO induced lower expression of miR156, which may positively regulate anthocyanin biosynthesis by raising the expression of SPL transcription factor. This is consistent with the results of our previous physiological experiments, and exogenous nitric oxide indeed promoted the synthesis of anthocyanins in alfalfa leaves in response to drought stress.

Exogenous NO may decrease due to the expression of miR399 in alfalfa to enhance drought tolerance. Plants suffering from water-deficiency usually show reduced uptake of mineral nutrients, and miR399 is involved in the regulation of phosphate $(\mathrm{P})$ homeostasis [58]. It has been described that miR399 has a negative regulation with inorganic phosphate concentration by targeting a pyrophosphatase. Most researches have shown that AVP1 (Arabidopsis vacuolar pyrophosphatase gene) is overexpressed in a variety of plants, with enhanced drought and salt tolerance, such as Arabidopsis and rice [59,60]. Consistent with previous findings in sugarcane [61], the current study results showed that the expression of miR399 family was decreased in the alfalfa under drought stress. Exogenous nitric oxide, which inhibits the expression of mtr-mir399a /c/q, can also increase pyrophosphatase levels and promote the proton pump activity. As it promotes proton pump activity, the water potential in the plant vacuole can lower the activity of secondary transporters. In addition, this can promote the flow of ions in the cytoplasm [61]. The present results reported changes in the transporter activity.

The researchers of the present study speculated that exogenous NO may regulate the expression of miRNA398 to eliminate ROS produced by drought stress. mtr-miR398a-5p is an important member of the miR398 family. The miR398 family in Medicago sativa was up-regulated under drought stress, and resembled the results found in Convolvulaceae, Medicago truncatula, and Jacquemontia 
pentantha [62-64], but differed from the results reported in Ipomoea campanulata [65]. Previous studies have shown that miR398 was crucial for plant stress responses, as it regulates copper-zinc superoxide dismutase (CuZnSOD) levels [66]. Similarly, the present study detected that miR398b targeted UDP-glucosyltransferase (UGT) HRA25 to enable drought stress response. Reduction in the expression level of mtr-miR398a-5p causes increase in the activity of CuZnSODs by achieving the oxidation protection of plants [65]. Exogenous NO silenced the expression of mtr-mir398a-5p, which consequently induced CuZnSODs. Physiological studies have explained that exogenous NO can remove ROS by increasing the activity of SOD [17].

Under drought stress, exogenous NO induced or silenced some conserved miRNAs, which regulate some novel miRNAs. unconservative_c109154.graph_c1_8010 and unconservative_c121624.graph_c0_25405 were down-regulated by exogenous NO, and participated in the metabolic and oxidation-reduction process targeting peroxisome and L-ascorbate oxidase. The novel miRNAs results contribute to further understanding of the regulatory mechanism of exogenous NO under drought stress, as well as supplementing the existing miRNAs. GO enrichment and KEGG pathway analysis were conducted on the target genes of differentially expressed miRNA to further clarify their functions and pathways involved in alfalfa's response to drought stress. Water shortages along with hydrogen peroxide and respiratory burst were involved in defense response and carbohydrate transport. These were significantly found and have confirmed to be associated with abiotic stresses in many plants [67]. Existing reports confirm that abscisic acid is involved in the metabolism of hydrogen peroxide $\left(\mathrm{H}_{2} \mathrm{O}_{2}\right)$ and calcium-dependent protein kinase (AtCPK1) to improve the drought resistance of plants [67,68]. Drought responses of carbohydrate metabolism may be important for plant acclimation to stress-this was later verified in Oryza sativa [53].

This study reported that carbohydrate transport was significantly enriched in alfalfa under drought stress and that their target genes were positively regulated. Accelerated carbohydrate transport can promote the distribution of carbohydrates in cells and tissues to aid in improving the drought resistance of alfalfa. It is worth mentioning that the metabolic pathways of starch and sucrose (ko00500) have also been enriched in this study. Other studies have shown that inhibition of starch synthesis can directly lead to an increase in soluble sugars of sucrose and glucose $[69,70]$. The rapid increase of soluble sugars, such as sucrose, in plant cells can form a vitreous state to prevent cell collapse, limiting the mixture of macromolecules in a stable and static state of cells [71]. The present results explain that the exogenous nitric oxide can induce more biological processes to enable participation in the drought resistance of alfalfa. It was further confirmed that exogenous nitric oxide induced more biological processes for active participation in the drought resistance of alfalfa. Among them, mtr-miR5752a was particularly induced and up-regulated by exogenous NO under drought stress, and its target gene could regulate negatively by starch biosynthetic (GO:0019252) and amylopectin biosynthetic process (GO:0010021) to cope with drought stress. This provides important evidence and ideas for the study of the effects of exogenous $\mathrm{NO}$ on alfalfa drought resistance.

\section{Conclusions}

A total of 90 discovered miRNAs and 177 unknown miRNAs were found in this experiment. The 14 discovered miRNAs and 18 uncharted miRNAs in the drought response of alfalfa further supplemented the drought-responsive miRNAs. The results provide a lucid understanding of the drought response mechanism of alfalfa and further explain the theoretical basis and technical support for molecular breeding and genetic improvement of alfalfa. In total, 24 known miRNAs and 31 novel miRNAs responded to exogenous nitric oxide under drought stress. Similar to the target genes, the researchers indicated the function of multiple miRNAs by GO enrichment and KEGG analysis. Thus far, this research is the first to investigate the mechanism of exogenous NO regulating plant drought-resistant miRNAs under drought stress, and also provides a better understanding for the action of the exogenous NO mechanism and its application. Simultaneously, the study has reported 
important scientific value and practical significance for revealing the method of exogenous nitric oxide improving plant drought stress resistance.

Supplementary Materials: The following are available online at http://www.mdpi.com/2073-4425/11/1/30/s1: Table S1: Sequences of miRNA and target genes primer. Table S2: The composition and expression of known miRNAs in alfalfa detected by CK, PEG, and PEG + SNP. Table S3: Composition and read amount (transcript per million (TPM) value) of known miRNAs in alfalfa detected under the three treatment conditions of CK, PEG, and PEG + SNP. Table S4: Maturation and precursor sequences and other information for predicted miRNA under three treatments, including read range, genomic ID, miRNA length, and FPKM. Table S5: New miRNA members and their readings treated with CK, PEG and PEG + SNP. Table S6: Expression abundance and detailed information of drought stress response miRNA, including FPKM, log fold-change, and $p$-value. Table S7: Expression abundance and detailed information of exogenous NO responsive miRNAs under drought stress, including FPKM, log fold-change, and $p$-value. Table S8: Prediction of differentially expressed miRNA target genes in alfalfa under drought stress. Table S9: Prediction of exogenous nitric oxide-responsive miRNA target genes under drought stress in alfalfa. Table S10: Some differentially expressed miRNA target gene information in PEG and PEG + SNP samples (target genes are SBP, Far-Redimpaired Response1 (FAR1), MYB and other transcription factors). Table S11: Functional annotation of NO-responsive miRNA target genes under drought-response and drought stress in alfalfa (gene ontology and KEGG pathway analyses). Table S12: Details of drought-responsive miRNA target gene enrichment classification (including biological processes, molecular functions, and cellular components). Table S13: Details of NO-responsive miRNA target gene enrichment classification under drought stress (including biological processes, molecular functions, and cellular components). Table S14: Information of KEGG pathway from PEG and PEG + SNP treatment.

Author Contributions: X.W. and W.M. conceived and designed the experiments. W.M., Y.Z. (Yaodong Zhao), and Y.Z. (Ying Zhao) performed the experiments. W.M., Y.L., Y.Z. (Yaodong Zhao), Q.L., and M.S. analyzed the data. W.M. and Y.Z. (Yaodong Zhao) wrote and modified the paper. All authors have read and agreed to the published version of the manuscript.

Funding: This work was supported by the National Natural Sciences Foundation of China (grant no. 31560663).

Acknowledgments: We thank James Rodriguez Rubio for his help with this article in English.

Conflicts of Interest: The authors declare that they have no competing interests.

\section{References}

1. Shu, Y.; Li, W.; Zhao, J.; Liu, Y.; Guo, C. Transcriptome sequencing and expression profiling of genes involved in the response to abiotic stress in Medicago ruthenica. Genet. Mol. Biol. 2018, 41, 638-648. [CrossRef] [PubMed]

2. Adhikari, L.; Lindstrom, O.M.; Markham, J.; Missaoui, A.M. Dissecting key adaptation traits in the polyploid perennial medicago sativa using GBS-SNP mapping. Front. Plant Sci. 2018, 9, 934-953. [CrossRef] [PubMed]

3. Liu, D.; Liu, M.; Liu, X.L.; Cheng, X.G.; Liang, Z.W. Silicon priming created an enhanced tolerance in alfalfa (Medicago sativa L.) seedlings in response to high alkaline stress. Front. Plant Sci. 2018, 9, 716-727. [CrossRef] [PubMed]

4. Wang, Y.; Ren, W.; Li, Y.; Xu, Y.; Teng, Y.; Christie, P.; Luo, Y. Nontargeted metabolomic analysis to unravel the impact of di(2-ethylhexyl) phthalate stress on root exudates of alfalfa (Medicago sativa). Sci. Total Environ. 2019, 646, 212-219. [CrossRef] [PubMed]

5. Tang, L.; Cai, H.; Ji, W.; Luo, X.; Wang, Z.; Wu, J.; Wang, X.; Cui, L.; Wang, Y.; Zhu, Y.; et al. Overexpression of GsZFP1 enhances salt and drought tolerance in transgenic alfalfa (Medicago sativa L). Plant Physiol. Biochem. 2013, 71, 22-30. [CrossRef]

6. Arshad, M.; Feyissa, B.A.; Amyot, L.; Aung, B.; Hannoufa, A. MicroRNA156 improves drought stress tolerance in alfalfa (Medicago sativa) by silencing SPL13. Plant Sci. 2017, 258, 122-136. [CrossRef]

7. Xin, X.Q.; Wei, X.H.; Han, D.; Yue, K.; Zhao, Y. Study on the activities of antioxidant enzymes and isozymes of seeds of alfalfa germination under exogenous NO and reverse regulation of PEG stress. J. Grass Ind. 2018, 27, 105-112.

8. Wendehenne, D.; Pugin, A.; Klessig, D.F. Nitric oxide: Comparative synthesis and signaling in animal and plant cells. Trends Plant Sci. 2001, 6, 177-183. [CrossRef]

9. Procházková, D.; Haisel, D.; Wilhelmová, N. Effects of exogenous nitric oxide on photosynthesis. Photosynthetica 2013, 51, 483-489. [CrossRef] 
10. Tanou, G.; Job, C.; Rajjou, L.; Arc, E.; Belghazi, M.; Diamantidis, G.; Molassiotis, A.; Job, D. Proteomics reveals the overlapping roles of hydrogen peroxide and nitric oxide in the acclimation of citrus plants to salinity. Plant J. 2009, 60, 795-804. [CrossRef]

11. Hao, G.P.; Xing, Y.; Zhang, J.H. Role of nitric oxide dependence on nitric oxide synthase-like activity in the water stress signaling of maize seedling. J. Integr. Plant Biol. 2008, 50, 435-442. [CrossRef] [PubMed]

12. Singh, P.K.; Indoliya, Y.; Chauha, A.S.; Singh, S.P.; Singh, A.P.; Dwivedi, S.; Tripathi, R.D. Nitric oxide mediated transcriptional modulation enhances plant adaptive responses to arsenic stress. Sci. Rep. 2017, 7, 3592-3605. [CrossRef] [PubMed]

13. Chen, C.; Li, Q.; Wang, Q.; Lu, D.; Zhang, H.; Wang, J.; Fu, R. Transcriptional profiling provides new insights into the role of nitric oxide in enhancing Ganoderma oregonense resistance to heat stress. PLoS ONE 2017, 7, 15694-15708. [CrossRef] [PubMed]

14. Zhao, M.G.; Chen, L.; Zhang, L.L.; Zhang, W.H. Nitric reductase-dependent nitric oxide production is involved in cold acclimation and freezing tzaiolerance in Arabidopsis. Plant Physiol. 2009, 151, 755-767. [CrossRef] [PubMed]

15. Santisree, P.; Bhatnagar-Mathur, P.; Sharma, K.K. NO to drought-multifunctional role of nitric oxide in plant drought: Do we have all the answers? Plant Sci. 2015, 239, 44-55. [CrossRef] [PubMed]

16. Yi, Q.; Wei, X.H.; Qiang, X.; Zhao, Y.; Ding, C.F. Investigation into the mechanism of Mo-mediated $\mathrm{Ca}^{2+}$ signaling During seed Germination and antioxidation in Medicago sativa under drought stress. Acta Prataculturae Sin. 2016, 25, 57-65.

17. Xin, X.Q.; Wei, X.H.; Han, T.; Yue, K.; Zhao, Y. Research on exogenous NO and reverse regulation to the dynamic changes of antioxidant enzymes and isoenzymes in alfalfa seed germination under PEG stress. Acta Prataculturae Sin. 2018, 27, 105-112.

18. Bartel, D.P. MicroRNAs: Genomics, biogenesis, mechanism, and function. Cell 2004, 116, 281-297. [CrossRef]

19. Budak, H.; Akpinar, B.A. Plant miRNAs: Biogenesis, organization and origins. Funct. Integr. Genom. 2015, 15, 523-531. [CrossRef]

20. Kidner, C.A.; Martienssen, R.A. The developmental role of microRNA in plants. Curr. Opin. Plant Biol. 2005, 8, 38-44. [CrossRef]

21. Bai, B.; Shi, B.; Hou, N.; Cao, Y.; Meng, Y.; Bian, H.; Zhu, M.; Han, N. microRNAs participate in gene expression regulation and phytohormone cross-talk in barley embryo during seed development and germination. BMC Plant Biol. 2017, 17, 150-166. [CrossRef] [PubMed]

22. Rhoades, M.W.; Reinhart, B.J.; Lim, L.P.; Burge, C.B.; Bartel, B.; Bartel, D.P. Prediction of plant microRNA targets. Cell 2005, 110, 513-520. [CrossRef]

23. Chen, X. A MicroRNA as a translational repressor of APETALA2 in arabidopsis flower development. Science 2004, 303, 2022-2025. [CrossRef] [PubMed]

24. Palatnik, J.F.; Allen, E.; Wu, X.L.; Schommer, C.; Schwab, R.; Carrington, J.C. Control of leaf morphogenesis by micrornas. Nature 2003, 425, 257-263. [CrossRef] [PubMed]

25. Li, H.; Peng, T.; Wang, Q.; Wu, Y.; Chang, J.; Zhang, M.; Tang, G.; Li, C. Development of incompletely fused carpels in maize ovary revealed by miRNA, target gene and phytohormone analysis. Front. Plant Sci. 2017, 8, 463-480. [CrossRef]

26. Liu, M.M.; Yu, H.Y.; Zhao, G.J.; Huang, Q.F.; Lu, Y.G.; Ouyang, B. Identification of drought-responsive microRNAs in tomato using high-throughput sequencing. Funct. Integr. Genom. 2018, 18, 67-78. [CrossRef]

27. Zhang, N.; Yang, J.W.; Wang, Z.; Wen, Y.K.; Wang, J.; He, W.H.; Liu, B.L.; Si, H.J.; Wang, D. Identification of novel and conserved microRNAs related to drought stress in potato by deep sequencing. PLoS ONE 2014, 9, e95489. [CrossRef]

28. Dong, Z.H.; Zhang, J.H.; Zhu, Q.Z.; Zhao, L.F.; Sui, S.X.; Li, Z.S.; Zhang, Y.L.; Wang, H.; Tian, D.L.; Zhao, Y.K. Identification of microRNAs involved in drought stress responses in early-maturing cotton by high-throughput sequencing. Genes Genom. Genes Genom. 2018, 40, 305-314. [CrossRef]

29. Wang, T.Z.; Chen, L.; Zhao, M.G.; Tian, Q.Y.; Zhang, W.H. Identification of drought-responsive microRNAs in Medicago truncatula by genome-wide high-throughput sequencing. BMC Genom. 2011, 12, 367-378. [CrossRef]

30. Li, Y.; Wan, L.Q.; Bi, S.Y.; Wan, X.F.; Li, Z.Y.; Cao, J.; Tong, Z.Y.; Xu, H.Y.; He, F.; Li, X.L. Identification of drought-responsive MicroRNAs from roots and leaves of alfalfa by high-throughput sequencing. Genes 2017, 8, 119. [CrossRef] 
31. Arshad, M.; Gruber, M.Y.; Hannoufa, A. Transcriptome analysis of microRNA156 overexpression alfalfa roots under drought stress. Sci. Rep. 2018, 8, 9363-9376. [CrossRef] [PubMed]

32. Long, R.C.; Li, M.N.; Kang, J.M.; Zhang, T.J.; Sun, Y.; Yang, Q.C. Small RNA deep sequencing identifies novel and salt-stress-regulated microRNAs from roots of Medicago sativa and Medicago truncatula. Physiol. Plant 2015, 154, 13-27. [CrossRef] [PubMed]

33. Fan, W.N.; Zhang, S.H.; Du, H.Q.; Sun, X.G.; Shi, Y.H.; Wang, C.Z. Genome-wide identification of different dormant Medicago sativa L. MicroRNAs in response to fall dormancy. PLoS ONE 2014, 9, e114612. [CrossRef] [PubMed]

34. Shu, Y.J.; Liu, Y.; Li, W.; Song, L.L.; Zhang, J.; Guo, C.H. Genome-wide investigation of MicroRNAs and their targets in response to freezing stress in Medicago sativa L. Based on High-Throughput Sequencing. G3 2016, 6, 755-765. [CrossRef] [PubMed]

35. Li, Z.Y.; Xu, H.Y.; Li, Y.; Wan, X.F.; Ma, Z.; Cao, J.; Li, Z.S.; He, F.; Wang, Y.F.; Wan, L.Q.; et al. Analysis of physiological and miRNA responses to Pi deficiency in alfalfa (Medicago sativa L.). Plant Mol. Biol. 2018, 96, 473-492. [CrossRef]

36. Cui, L.G.; Shan, J.X.; Shi, M.; Gao, J.P.; Lin, H.X. The miR156-SPL9-DFR pathway coordinates the relationship between development and abiotic stress tolerance in plants. Plant J. 2014, 80, 1108-1117. [CrossRef]

37. Saminathan, T.; Bodunrin, A.; Singh, N.V.; Devarajan, R.; Nimmakayala, P.; Jeff, M.; Aradhya, M.; Reddy, U.K. Genome-wide identification of microRNAs in pomegranate (Punica granatum L.) by high-throughput sequencing. BMC Plant Biol. 2016, 16, 122. [CrossRef]

38. Liu, H.H.; Tian, X.; Li, Y.J.; Wu, C.A.; Zheng, C.C. Microarray-based analysis of stress-regulated microRNAs in Arabidopsis thaliana. RNA 2008, 14, 836-843. [CrossRef]

39. Lopez-Arredondo, D.L.; Leyva-Gonzalez, M.A.; González-Morales, S.I.; López-Bucio, J.; Herrera-Estrella, L. Phosphate nutrition:improving low-phosphate tolerance in crops. Annu. Rev. Plant Biol. 2014, 65, 95-123. [CrossRef]

40. Cao, C.Y.; Long, R.C.; Zhang, T.J.; Junmei Kang, J.M.; Wang, Z.; Wang, P.Q.; Sun, H.; Yu, J.; Yang, Q.C. Genome-wide identification of microRNAs in response to salt/alkali stress in Medicago truncatula through high-throughput sequencing. Int. J. Mol. Sci. 2018, 19, 4076. [CrossRef]

41. Devers, E.A.; Branscheid, A.; May, P.; Krajinski, F. Stars and symbiosis: microRNA- and microRNA-mediated transcript cleavage involved in arbuscular mycorrhizal symbiosis. Plant Physiol. 2011, 156, 1990-2010. [CrossRef] [PubMed]

42. Zhai, J.; Jeong, D.H.; De, P.E.; Park, S.; Rosen, B.D.; Li, Y.; González, A.J.; Yan, Z.; Kitto, S.L.; Grusak, M.A.; et al. MicroRNAs as master regulators of the plant NB-LRR defense gene family via the production of phased, trans-acting siRNAs. Genes Dev. 2011, 25, 2540-2553. [CrossRef] [PubMed]

43. Eyles, R.P.; Williams, P.H.; Ohms, S.J.; Weiller, G.F.; Ogilvie, H.A.; Djordjevic, M.A.; Imin, N. microRNA profiling of root tissues and root forming explant cultures in Medicago truncatula. Planta 2013, 238, 91-105. [CrossRef] [PubMed]

44. Cohu, C.M.; Abdel-Ghany, S.E.; Gogolin-Reynolds, K.A.; Onofrio, A.M.; Bodecker, J.R.; Kimbrel, J.A.; Niyogi, K.K.; Pilon, M. Copper Delivery by the Copper Chaperone for Chloroplast and Cytosolic Copper/Zinc-Superoxide Dismutases: Regulation and Unexpected Phenotypes in an Arabidopsis Mutant. Mol. Plant 2009, 2, 1336-1350. [CrossRef] [PubMed]

45. Gao, J. Genome-wide identification and function analysis of SBP gene family in maize. Acta Agron. Sin. 2016, 42, 201-211.

46. Liu, J.; Cheng, X.L.; Liu, P.; Sun, J.Q. miR156-Targeted SBP-Box transcription factors interact with DWARF53 to regulate TEOSINTE BRANCHED1 and BARREN STALK1 expression in bread wheat. Plant Physiol. 2017, 174, 1931-1948. [CrossRef]

47. Dubos, C.; Stracke, R.; Grotewold, E.; Weisshaar, B.; Martin, C.; Lepiniec, L. MYB transcription factors in arabidopsis. Trends Plant Sci. 2010, 15, 573-581. [CrossRef]

48. Roy, S. Function of $M Y B$ domain transcription factors in abiotic stress and epigenetic control of stress response in plant genome. Plant Signal. Behav. 2016, 11, e1117723. [CrossRef]

49. Liu, J.Y.; Osbourn, A.; Pengda, M. MYB transcription factors as regulators of phenylpropanoid metabolism in plants. Mol. Plant 2015, 8, 689-708. [CrossRef]

50. Castroverde, C.D.M. The AREB1-ADA2b-GCN5 complex regulates gene expression during drought stress. Plant Cell 2018, 31, 559-560. [CrossRef] 
51. Batlang, U.; Baisakh, N.; Ambavaram, M.M.; Pereira, A. Phenotypic and physiological evaluation for drought and salinity stress responses in rice. Methods Mol. Biol. 2013, 956, 209-225. [PubMed]

52. Mohammadi, P.P.; Moieni, A.; Komatsu, S. Comparative proteome analysis of drought-sensitive and drought-tolerant rapeseed roots and their hybrid F1 line under drought stress. Amino Acids 2012, 43, 2137-2152. [CrossRef] [PubMed]

53. Chen, J.J.; Li, L.Z. Multiple regression analysis reveals microRNA regulatory networks in oryza sativa under drought stress. Int. J. Genom. 2018, 2018, 9395261. [CrossRef] [PubMed]

54. Xu, Y.J.; Zhu, S.W.; Liu, F.; Wang, W.; Wang, X.W.; Han, G.M.; Cheng, B.J. Identification of arbuscular mycorrhiza fungi responsive microRNAs and their regulatory network in maize. Int. J. Mol. Sci. 2018, 19, 3201. [CrossRef] [PubMed]

55. Zhou, Z.S.; Zeng, H.Q.; Liu, Z.P.; Yang, Z.M. Genome-wide identification of Medicago truncatula microRNAs and their targets reveals their differential regulation by heavy metal. Plant Cell Environ. 2012, 35, 86-99. [CrossRef] [PubMed]

56. Candar-Cakir, B.; Arican, E.; Zhang, B. Small RNA and degradome deep sequencing reveals drought-and tissue-specific micrornas and their important roles in drought-sensitive and drought-tolerant tomato genotypes. Plant Biotechnol. J. 2016, 14, 1727-1746. [CrossRef] [PubMed]

57. Kim, H.E.; Kim, W.; Lee, A.R.; Jin, S.; Jun, A.R.; Kim, N.K.; Lee, J.H.; Ahn, J.H. Base-pair opening dynamics of the microRNA precursor pri-miR156a affect temperature-responsive flowering in Arabidopsis. Biochem. Biophys. Res. Commun. 2017, 484, 839-844. [CrossRef]

58. Wang, Y.; Zhang, J.X.; Cui, W.X.; Guan, C.Y.; Mao, W.J.; Zhang, Z.H. Improvement of fruit quality by overexpressing miR399 in woodland strawberry. J. Agric. Food Chem. 2017, 65, 7361-7370. [CrossRef]

59. Gaxiola, R.A.; Li, J.; Undurraga, S.; Dang, L.M.; Allen, G.J.; Alper, S.L.; Fink, G.R. Drought- and salt-tolerant plants result from overexpression of the AVP1 H+-pump. Proc. Natl. Acad. Sci. USA 2001, 98, 11444-11449. [CrossRef]

60. Zhao, F.Y.; Zhang, X.J.; Li, P.H.; Zhao, Y.X.; Zhang, H. Co-expression of thesuaeda salsa ssnhx1andarabidopsis avp1confer greater salt tolerance to transgenic rice than the singleSsnhx1. Mol. Breed. 2006, 17, 341-353. [CrossRef]

61. Ferreira, T.H.; Gentile, A.; Vilela, R.D.; Costa, G.G.; Dias, L.I.; Endres, L.; Menossi, M. microRNAs associated with drought response in the bioenergy crop sugarcane (Saccharum spp.). PLoS ONE 2012, 7, e46703. [CrossRef] [PubMed]

62. Ghorecha, V.; Zheng, Y.; Liu, L.; Sunkar, R.; Krishnayya, N.S.R. MicroRNA dynamics in a wild and cultivated species of Convolvulaceae exposed to drought stress. Physiol. Mol. Biol. Plants 2017, 23, 291-300. [CrossRef] [PubMed]

63. Trindade, I.; Capitao, C.; Dalmay, T.; Fevereiro, M.; Santos, D. miR398 and miR408 are up-regulated in response to water deficit in Medicago truncatula. Planta 2010, 231, 705-716. [CrossRef] [PubMed]

64. Ghorecha, V.; Patel, K.; Ingle, S.; Sunkar, R.; Krishnayya, N.S. Analysis of biochemical variations and microRNA expression in wild (Ipomoea campanulata) and cultivated (Jacquemontia pentantha) species exposed to in vivo water stress. Physiol. Mol. Biol. Plants 2014, 20,57-67. [CrossRef]

65. Sunkar, R.; Zhu, J.K. Novel and stress-regulated microRNAs and other small RNAs from Arabidopsis. Plant Cell 2004, 16, 2001-2019. [CrossRef] [PubMed]

66. Sunkar, R.; Kapoor, A.; Zhu, J.K. Posttranscriptional induction of two $\mathrm{Cu} / \mathrm{Zn}$ superoxide dismutase genes in Arabidopsis is mediated by downregulation of miR398 and important for oxidative stress tolerance. Plant Cell 2006, 18, 2051-2065. [CrossRef] [PubMed]

67. Li, C.X.; Bian, B.T.; Gong, T.Y.; Liao, W.B. Comparative proteomic analysis of key proteins during abscisic acid-hydrogen peroxide-induced adventitious rooting in cucumber (Cucumis sativus L.) under drought stress. J. Plant Physiol. 2018, 229, 185-194. [CrossRef]

68. Huang, K.; Peng, L.; Liu, Y.Y.; Yao, R.D.; Liu, Z.B.; Li, X.F.; Yang, Y.; Wang, J.M. Arabidopsis calcium-dependent protein kinase AtCPK1 plays a positive role in salt/drought-stress response. Biochem. Biophys. Res. Commun. 2018, 498, 92-98. [CrossRef]

69. Geigenberger, P.; Stitt, M. Diurnal changes in sucrose, nucleotides, starch synthesis and AGPS transcript in growing potato tubers that are suppressed by decreased expression of sucrose phosphate synthase. Plant J. 2000, 23, 795-806. [CrossRef] 
70. Tiessen, A.; Prescha, K.; Branscheid, A.; Palacios, N.; McKibbin, R.; Halford, N.G.; Geigenberger, P. Evidence that SNF1-related kinase and hexokinase are involved in separate sugar-signaling pathways modulating post-translational redox activation of ADP-glucose pyrophosphorylase in potato tubers. Plant J. 2003, 35, 490-500. [CrossRef]

71. Koster, K.L. Glass formation and desiccation tolerance in seeds. Plant Physiol. 1996, 96, 302-304. [CrossRef]

(C) 2019 by the authors. Licensee MDPI, Basel, Switzerland. This article is an open access article distributed under the terms and conditions of the Creative Commons Attribution (CC BY) license (http://creativecommons.org/licenses/by/4.0/). 